\title{
Unbiased Stereology applied to Microscopic Investigation of Human Recurrent Laryngeal Nerve Epineurium
}

\section{Guowei Xia, Julie Barkmeier-Kraemer, Kang Li, Michelle Ciucci}

Department of Speech, Language, and Hearing Sciences, the University of Arizona, Tucson, AZ 85721

The purpose of this poster is to describe the methodology used to investigate the connective tissue composition of the epineurium in the recurrent laryngeal nerve (RLN) branch of the vagus nerve.

Light microscopy montages: Each of 10 bilateral RLNs was excised in entirety from its origin at the vagus nerve to its insertion into the posterior larynx (see Figure 1a). Due to the longer length of the left RLN, it was divided into 6 segments and the right RLN was divided into 4 segments that were approximately $2-\mathrm{cm}$ in length. All segments were embedded in paraffin, cut into 10 micron cross-sections, and stained with Mallory's Trichrome. Light microscopy images were digitized at $200 \mathrm{X}$ with a SPOT color digital camera attached to a Nikon T-200 bright-field microscope. Due to the diameter of the RLNs, several image files were digitized associated with each nerve section. These were then spliced into montages of the entire nerve cross-section for each segment of the nerve using Adobe ${ }^{\circledR}$ Photoshop (Fig.1b).

Stereologic Analysis: The point-to-point method of unbiased stereology ${ }^{[3]}$ was used to determine the RLN volume associated with the components of the epineurium. This was achieved by manually placing boundaries around the epineurium and nerve fascicles of each image using Adobe $^{\circledR}$ Photoshop. Next, a transparent image of calibrated grids generated by the MatLab ${ }^{\circledR}$ software was overlaid on each cross-sectional image (Fig.2). The grid numbers were manually counted using a MatLab ${ }^{\circledR}$ program interface to determine the total area of the epineurium, and the proportion of each RLN section's epineurium composed of adipose, collagen, and blood vessels. The volumes and relative proportions of epineurium and its connective tissue components were calculated using the Cavalieri principle.

Conclusion: This methodology allowed cross-sectional analysis of the RLN at an adequate magnification for visualization and measurement of the connective tissues of the epineurium. Further, the point-to-point method of unbiased stereology using MatLab ${ }^{\circledR}$ allowed minimal error and calculation of a volumetric estimate necessary for future biomechanical testing of the nerve.

\section{References:}

[1] Benninger, Gillen, \& Altman. Laryngoscope. 1998; 108(9):1346

[2] Barkmeier and Luschei. J. Anat. 2000; 196(1):85

[3] Gundersen. J Microsc. 1986; 143 (Pt 1):3 

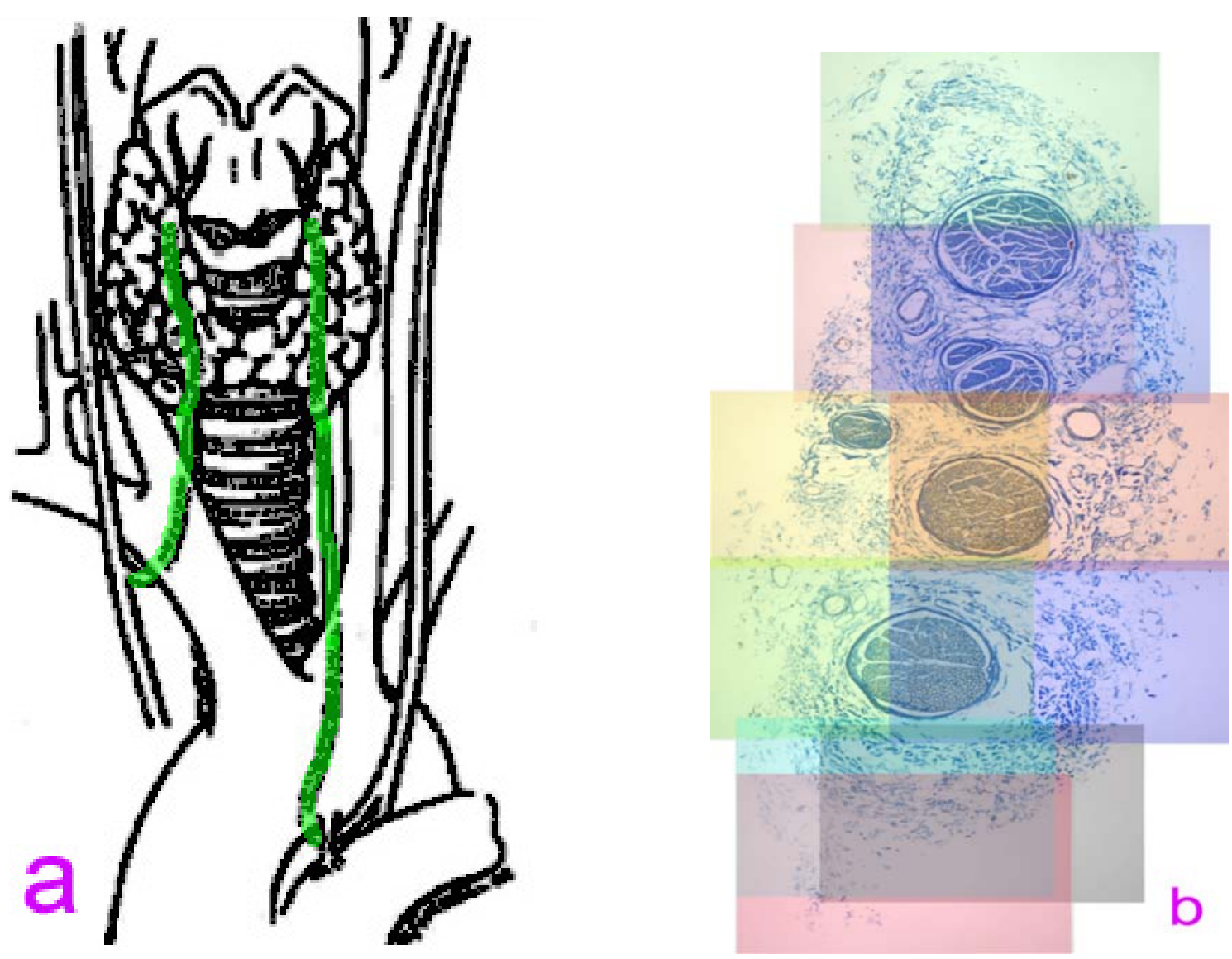

Fig.1 a. The RLN excised for study (in green). b. The montage of the cross-sectional images of the RLN (200X). Different colors were overlaid on each single image.
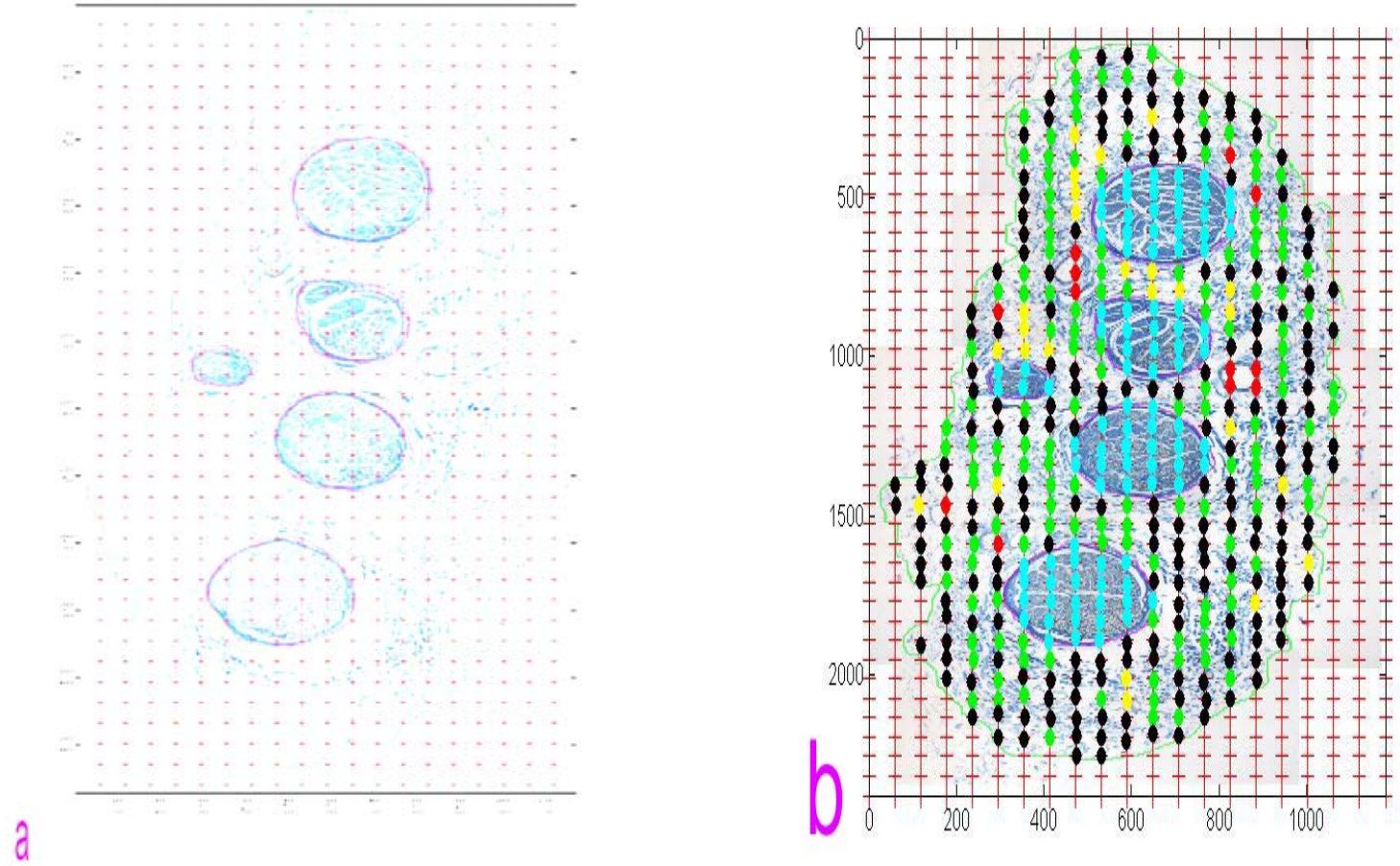

Fig.2 a. Transparent grids were overlaid on the cross-sectional image of the RLN. b. The grids were counted to estimate the volumes and relative proportions of epineurium and its connective tissue components. 